\title{
Evaluation of working length determination based on the analysis of cone-beam computed tomographic images and an electronic apex locator: a retrospective study*
}

\author{
Krystyna PietrzyckaA , Mateusz Radwański ${ }^{\circledR}$, Halina Pawlicka ${ }^{\mathrm{C}}$ \\ Medical University of Lodz, Department of Endodontics, Pomorska 251, 92-213 Łódź, Poland

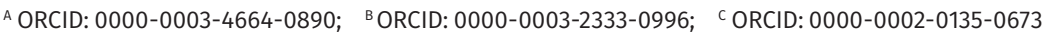 \\ $\triangle$ mateusz.radwanski@gmail.com
}

\begin{abstract}
Introduction: Determining the length of a root canal is a significant outcome predictor for endodontic treatment. The aim of this retrospective, clinical study was to evaluate the accuracy of working length (WL) determination using pre-existing conebeam computed tomography (CBCT) images and to compare them with data of the clinical WL determined with an electronic apex locator (EAL) in treated teeth, found in patient records.

Materials and methods: Thirty five medical records of patients who underwent root canal treatment (RCT) by an endodontist in a private dental practice in Łódź, Poland were analysed. Evaluations were conducted on the data concerning: the WL of the treated tooth as found in the patient's records, the RadioVisioGraphy (RVG) image after final obturation used as a control, and the CBCT scan before RCT. The Shapiro-Wilk normality
\end{abstract}

test and Student's t-test were used in statistical analysis. The significance level was set at $\mathrm{p}<0.05$.

Results: There were no statistically significant differences between compared groups in terms of WL determined with an EAL than CBCT ( $p>0.05$ ). In the case of medical diagnosis (pulpitis vs. necrotic pulp) and treatment (primary vs. retreatment), the obtained results were also statistically insignificant $(p>0.05)$. Conclusions: This retrospective, clinical study showed that CBCT scans can be successfully used for endodontic WL determination. Future investigations are needed to evaluate whether pre-existing CBCT scans may replace initial periapical radiographs (PARs).

Keywords: electronic apex locator; cone-beam computed tomography; root canal treatment; working length determination.

\section{INTRODUCTION}

The removal of infected pulp, necrotic debris and microorganisms from the root canal space is crucial for successful endodontic treatment. A successful obturation of a root canal prevents the healing of existing lesions in the periapical tissues and the development of any new ones. These goals can be achieved when the working length (WL) is assessed correctly. Overestimation of the WL may lead to over instrumentation of the apical region, perforation, and overfilling, whereas underestimation may cause insufficient canal system preparation, and thus, the development of periapical lesions. Numerous scientific studies have shown that the cementodentinal junction is the terminus point of root canal preparation and obturation. This is the narrowest point in the canal and is located 0.5-2 mm superior to the apical foramen. The major foramen is usually situated laterally on the mesial or distal side of the root. So far, dental practitioners have relied on periapical radiographs (PARs) and electronic apex locators (EALs) [1] to find this point. However, it has been reported that an open apex, apical periodontitis, the gauge of the major foramen, the size of the file used to assess the root canal length and the obliteration of the root canal can compromise the precision of the

EAL $[2,3]$. Similarly, radiographic measurements of WL can be problematic. The radiographic method of WL verification lacks accuracy because it is based on the average location of the cementodentinal junction. Periapical radiograph represents a 2-dimensional image of a 3-dimensional object and a distortion in the size and shape of the object is possible.

With the great technological advances of recent years, a new diagnostic tool known as the cone-beam computed tomography (CBCT) has been developed. A number of limitations that come with traditional radiographic images for WL determination have been overcome in 3-dimensional CBCT examinations. Recent studies have concluded that CBCT images are more sensitive than PARs in estimating root canal anatomy, detecting periapical lesions, and root fractures and perforations. Some scientific studies have compared the use of CBCT scans with EALs for WL determination in vivo [4, 5].

The aim of this retrospective, clinical examination was to evaluate the precision of WL measurements in pre-existing CBCT images and to compare them with data found in the patients' records where WL was established by using an EAL. The null hypothesis tested was that there was no difference between CBCT and EAL in WL determination. 


\section{MATERIALS AND METHODS}

\section{Sample selection}

After a local ethics committee granted approval (RNN/276/17/ $\mathrm{KE}), 120$ records of patients who were referred to a private dental practice for primary root canal therapy and retreatment were selected. The medical history of treated patients was irrelevant, and they all had pre-existing CBCT scans. No patient underwent a CBCT examination exclusively for this study. The CBCT scans had been performed for endodontic, periodontal, dental implant placement or surgical purposes. The inclusion criteria concerned single- or double-rooted teeth (incisors, canines, and premolars). Molar teeth with several canals, roots with external root resorption, an open apex or teeth with amalgam fillings were excluded from the study. Scans of low quality or with additional artefacts were also excluded from the examination. Therefore, the final study group included data from the medical records of 35 patients.

\section{Estimation of working length determination on the cone-beam computed tomography scans}

Examinations were uploaded using the iCAT Vision ${ }^{\circledR}$ scanner (Gendex, USA). Imaging parameters were $120 \mathrm{kVp}, 5.0 \mathrm{~mA}$, $0.125-0.25 \mathrm{~mm}$ voxel protocols with an exposure time of $7 \mathrm{~s}$. All scans were inspected with the use of specialized computer software (iCATVision Q, ver. 1.9.3.13; Gendex, USA).

The CBCT images were analyzed on an MSI Wind Top AE2220, LCD 21.5-inch screen with a resolution of $1920 \times 1080 \mathrm{px}$. The contrast and brightness of the images were altered with the use of an image-processing tool to provide optimal visualization. Two independent researchers (an endodontist and a general dental practitioner) estimated the length of all samples and any disagreement was discussed until a consensus was reached. For the final examination, each measurement was performed twice, with a 2-week break between each estimation. During the estimation of the WL measurements on the CBCT scans, each root canal was placed in a vertical position to visualize, whenever possible, the entire length of the canal in one single slice. The WL on CBCT scans was the distance between 2 points: the incisal or cuspidal edge for anterior and posterior teeth respectively and the radiographic apex (Fig. 1). Finally, the data received by researchers were averaged and mean values were calculated.

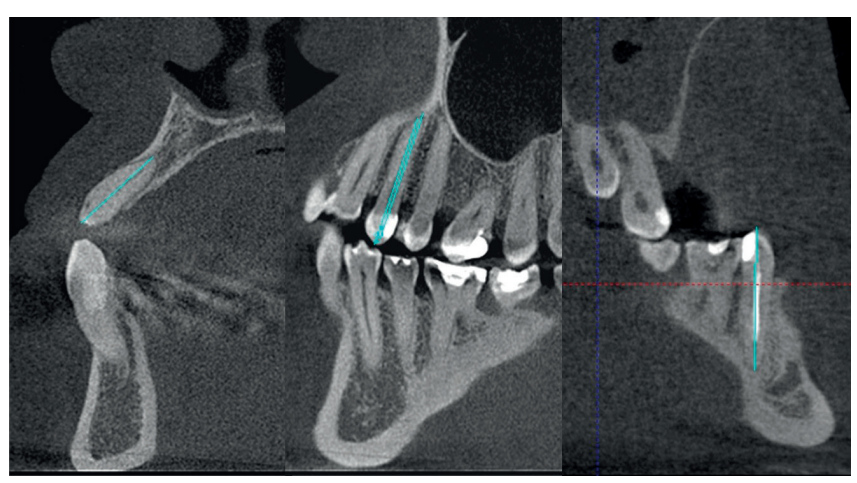

FIGURE 1. The establishment of root canal length in the cone-beam computed tomography (CBCT) images

\section{Analysis of medical records}

All root canal treatments (RCTs) were performed by an expert endodontist. The reference points from the multi-frequency EAL (Endopilot ${ }^{\circledR}$, Schlumbohm, Germany) used to establish the WL were the same as those used in the CBCT scans. All teeth were treated under aseptic conditions with a rubber-dam isolation, and the trepanation of the pulp cavity was carried out using an operating microscope until straight-line access to the canal was obtained. All measurements of WL were taken after irrigation with 5.25\% NaOCl. K-files \#15 (Dentsply Maillefer) connected to an EAL. The measurements were established following the manufacturer's instructions using an Endopilot apex locator. When the 0.0 signal was noticed, the file was moved beyond the apex until the sonic signal "beyond apex" was heard. The file was then removed until the 0.0 point. The distance was recorded by using Ruler Endodontics (VDW, Germany). All root canals were shaped to the 35/.04 Mtwo file (VDW, Germany). During the preparation, root canals were irrigated with 5.25\% $\mathrm{NaOCl}$, and finally rinsed with $15 \%$ EDTA and alcohol with silver nanoparticles (Nanocare ${ }^{\circledR}$ Plus, Dental Nanotechnology, Poland). Canals were filled using the continuous wave of condensation method and AHPlus ${ }^{\circledR}$ as a sealant (Dentsply, Switzerland). After the canals were filled, an RadioVisioGraphy (RVG) image was created (Fig. 2).

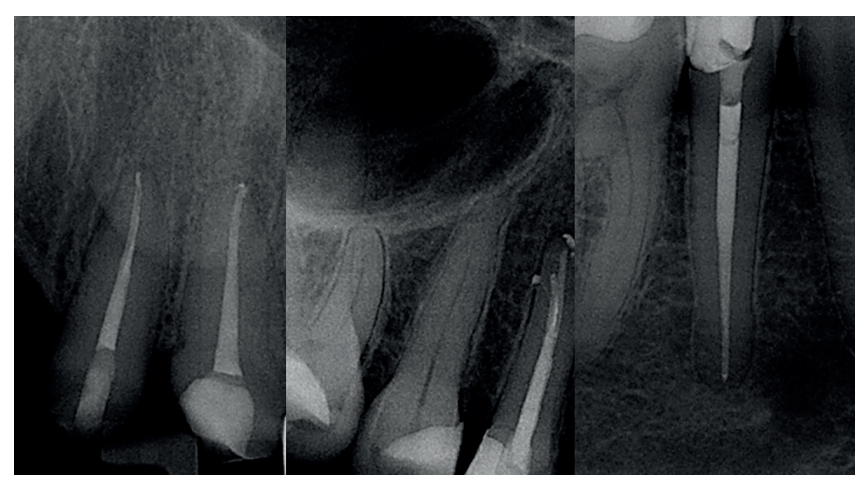

FIGURE 2. The RadioVisioGraphy (RVG) images after root canal treatment (RCT)

Evaluations were conducted on the data concerning the WL of the treated tooth as found in the patient's records, the control RVG image after final obturation, and the CBCT scan before RCT. The analysis was conducted in 2019.

\section{Statistical analysis}

Statistical analysis was performed in Statistica 13.1 PL (Statsoft, Polska). The normality distribution was checked using the Shapiro-Wilk normality test. The comparison between groups with a normal distribution was conducted with the Student's t-test. The level of statistical significance was set at $\mathrm{p}<0.05$.

\section{RESULTS}

The study group consisted of 35 patients: 22 men (62.58\%) and 13 women (37.15\%). The average age was $44.14 \pm 10.78$ years (max. 68 years and min. 21 years). The analysis involved 48 teeth: 13 
incisors (27.08\%), 5 canines (10.42\%) and 30 premolars (62.5\%). Altogether, 58 canals were examined. Radiographs of each case showed that all canals were filled properly.

Primary endodontic treatment was carried out in 28 cases (58.33\%), 15 of which were related to the diagnosis of vital pulp (53.57\%) and 13 to necrotic pulp (46.43\%). Retreatment was conducted on 20 teeth (41.67\%). Primary treatment was conducted on 34 canals (including 16 vital pulp and 18 necrotic pulp cases) - 58.62\% - and retreatment was conducted on 24 canals $(41.38 \%)$.

The data obtained from WL analysis are presented in the figures below (Fig. 3, 4, 5).

There was no statistically significant correlation between the compared groups in terms of the determination of WL with an EAL or CBCT ( $\mathrm{p}>0.05)$ reported. In the case of medical diagnosis (vital pulp vs. necrotic pulp) and treatment (primary vs. retreatment), the obtained results were also statistically insignificant $(p>0.05)$.

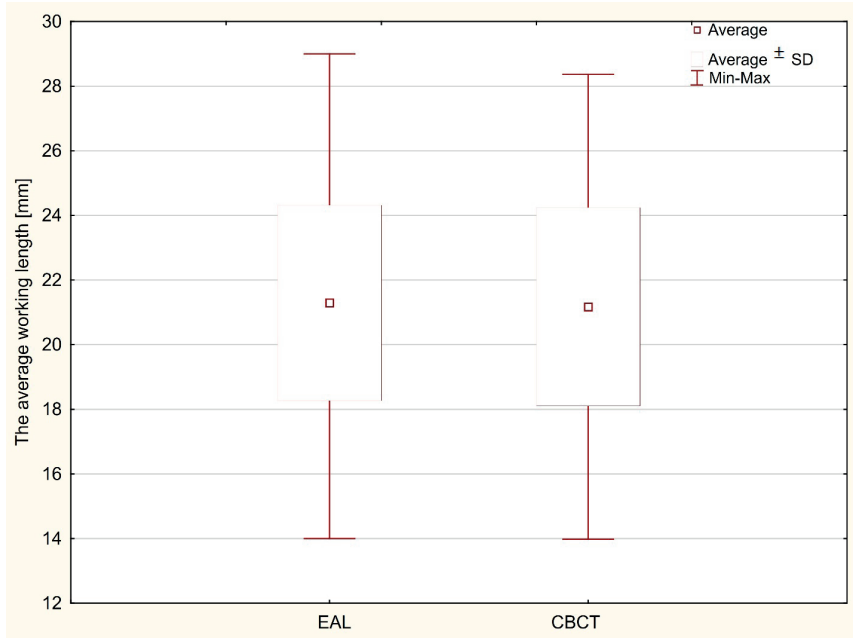

FIGURE 3. Comparison of the working length measurement with an electronic apex locator (EAL) and cone-beam computed tomography (CBCT)

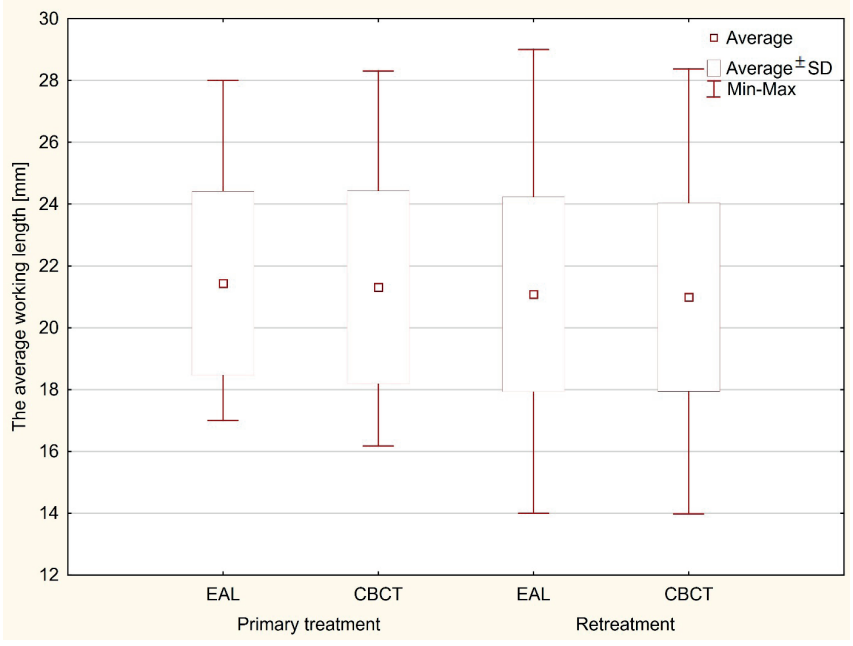

EAL - electronic apex locator; CBCT - cone-beam computed tomography

FIGURE 4. Comparison of the working length measurement in the case of primary treatment

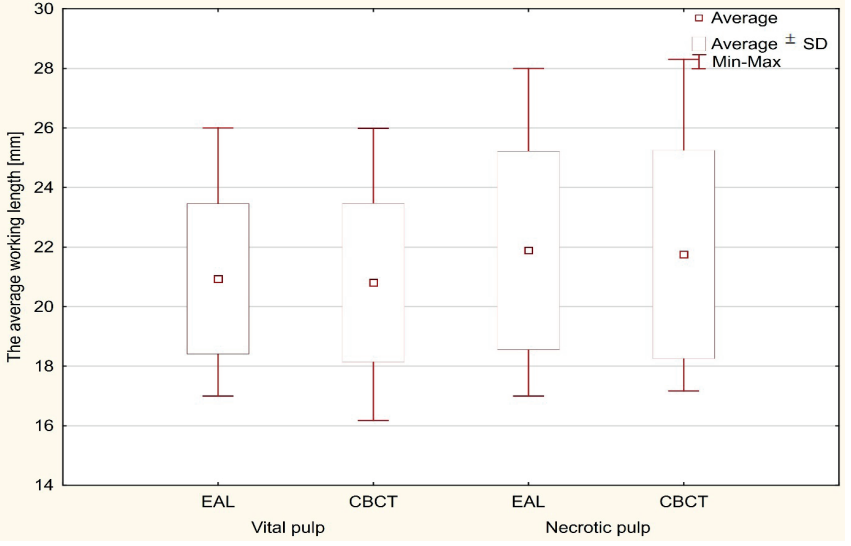

EAL - electronic apex locator; CBCT - cone-beam computed tomography

FIGURE 5. Comparison of the working length measurement in the case of teeth with vital and necrotic pulp

\section{DISCUSSION}

The objective of this retrospective, clinical study was to analyze clinical WL data obtained from pre-existing CBCT scans and to compare these with EAL estimations from patient records. The study showed that there were no significant differences in the measurements obtained through an EAL when compared to those obtained from CBCT scans. The null hypothesis can be accepted.

In previous studies, the measurement of the root canal length with EALs was conducted to different apical anatomical structures such as the cementodentinal junction/apical constriction or the major apical foramen/apical foramen [6, 7]. In our study, WL measurements with EALs were performed to the major foramen as the detection of cementodentinal junction/ apical constriction in CBCT images is impossible, even when the apical foramen is easily seen. For this reason, the major foramen, seen on CBCT images as the apical foramen, was used as the apical landmark for WL evaluation. This apical reference point was also applied by Üstün et al. [8]. In a study by Yılmaz et al., electronic measurements were established up until the apical foramen, however in CBCT scans, these were only established to the radiographically visible apex [9]. It should be mentioned that the coronal reference point on CBCT slices may not be identical to the actual cuspidal edge. The reference point may change in the case of teeth that have been excluded from occlusion during treatment or for those disturbed by metal fillings.

The results of this research are consistent with previous studies which evaluated the potential of CBCT in WL determination. Janner et al. found a high correlation between the effectiveness and accuracy of pre-existing CBCT images in measurements of the endodontic WL and standard clinical procedures [4]. In another in vivo study, de Morais et al.compared the accuracy of WL determination using CBCT scans, standard PARs and EALs. They found that WL measurements with the use of CBCT scans were more accurate when compared 
to radiographs and EALs although no statistically significant differences were observed between the 3 methods [10]. In their study, Ghule and Naik found no statistical differences between WL determination in primary teeth between the latest generation of EAL (CanalPro ${ }^{\circledR}$ ) and CBCT [11]. According to Jeger et al. limited CBCT images can be used for WL determination [5], this coincides with the observations of Mrasori et al. [12] and Dutta et al. [13].

In contrast, our findings conflict with results of other studies $[9,14]$ such as Lucena et al. who compared the precision of WL determination using the Raypex 6 EAL and CBCT scans [14] Methodological differences, such as voxel size, may have caused the discrepancy between the results of the studies. The observers estimated the CBCT scans with a voxel size of $0.5 \mathrm{~mm}$. Contrary to Lucena et al. [14], our findings were obtained using the iCAT Vision scanner with $0.125-0.25 \mathrm{~mm}$ voxel protocols.

Üstün et al. conducted a clinical study in which CBCT images were obtained preoperatively with a limited field of view and voxel size of $0.125 \mathrm{~mm}$. Seventy-three teeth in 30 patients were treated by an expert endodontist. Raypex ${ }^{\circledR} 6$ was used for determining the WL [8]. The results of the cited study and the results obtained from the present examination demonstrated that CBCT images can be used as an optional method for the measurement of WL. Metska et al., in an in situ study, compared the accuracy of root canal length determination in CBCT images using a voxel size of $0.125 \mathrm{~mm}$ with the real root canal length. Cone-beam computed tomography scans gave results comparable to the real root canal length [15].

Aktan et al. conducted a study which determined the effects of voxel size and resolution of CBCT on the measurement of WL. The evaluation of their data implies that CBCT scans can be useful for clinical WL measurement and that the smallest voxel size paired with the highest resolution will yield the most accurate results. Also, in the present investigation, EAL measurements were compared with measurements from preexisting CBCT images, and the received results were comparable [16]. In another study, Connert et al. evaluated the accuracy of endodontic WL measurements taken pre-operatively with a CBCT in 42 extracted human teeth. They found that the measurements of CBCT scans with a voxel size of $0.2 \mathrm{~mm}$ were not significantly different from the actual length [17]. In an ex vivo study, Yılmaz et al. evaluated the precision of WL estimation with the use of EAL, PARs, and CBCT scans performed with different voxel sizes $(0.080 \mathrm{~mm}, 0.125 \mathrm{~mm}, 0.160 \mathrm{~mm}$, $0.250 \mathrm{~mm}$ ) and fields of view in extracted human teeth. The results demonstrated that the $\mathrm{CBCT}$ images with different fields of view can be used for WL estimation [9].

It was also important to compare whether the status of the pulp (vital or necrotic), had a significant effect on the precision of EAL. Due to variations in the conditions of using apex locators in clinical practice, electronic measurements of the WL in canals with vital/necrotic pulp or during re-treatment are not included in the specifications for the EndoPilot ${ }^{\circledR}$. Although this was the case, it was relevant to include these experimental groups in our study. Similar to this investigation, several previous studies $[1,18]$ assessed whether pulp status had a significant effect on the precision of the EALs. Our results are analogous with the results of the cited reports and found that the precision of electronic WL measurement was not affected by the status of pulp tissue.

Our findings suggest that CBCT images ensure outcomes comparable with EALs for the confirmation of the endodontic WL. If a patient has a pre-existing CBCT scan, the dental practitioner should take advantage of this technique as an additional, reliable method for the determination of WL. Therefore, if a preoperative CBCT image is available, to conform with the rule of "as low as reasonably achievable" (ALARA), an additional preoperative X-ray should not be taken to avoid excessive radiographic exposure $[10,19]$.

However, there were some limitations of our study. The present study included a small group of single- or double-rooted teeth: incisors, canines, and premolars with straight canals and as such, measuring canal length using CBCT was uncomplicated. Further research should focus on the analysis of measurements in multi-rooted teeth (molars) and analyze the factors that may affect the measurements, such as the presence of periapical lesions, open apex, or the presence of resorption.

\section{CONCLUSIONS}

Within the limitations of our study, it can be stated that an existing CBCT image of teeth undergoing endodontic treatment can be useful for the endodontic WL determination. Taking an additional preoperative dental radiograph is unnecessary when the patient has a pre-existing CBCT scan. Any future prospective survey should estimate whether and when PARs, for the determination of endodontic WL, can be avoided when pre-existing CBCT scans are available.

\section{REFERENCES}

1. George R. Precision of multi-frequency electronic apex locators. Evid Based Dent 2016;17(3):86-7.

2. Razavian H, Mosleh H, Khazaei S, Vali A. Electronic apex locator: A comprehensive literature review - Part II: Effect of different clinical and technical conditions on electronic apex locator's accuracy. Dent Hypotheses 2014;5(4):133-141.

3. ElAyouti A, Dima E, Ohmer J, Sperl K, von Ohle C, Löst C. Consistency of apex locator function: a clinical study. J Endod 2009;35(2):179-81.

4. Janner SFM, Jeger FB, Lussi A, Bornstein MM. Precision of endodontic working length measurements: A pilot investigation comparing conebeam computed tomography scanning with standard measurement techniques. J Endod 2011;37(8):1046-51.

5. Jeger FB, Janner SFM, Bornstein MM, Lussi A. Endodontic working length measurement with preexisting cone-beam computed tomography scanning: A prospective, controlled clinical study. J Endod 2012;38(7):884-8.

6. Martins JNR, Marques D, Mata A, Caramês J. Clinical efficacy of electronic apex locators: Systematic review. J Endod 2014;40(6):759-77.

7. Chaudhary S, Gharti A, Adhikari B. An in vivo comparison of accuracy of two electronic apex locators in determining working length using stainless steel and nickel titanium files. Clin Cosmet Investig Dent 2018;10:75-82.

8. Üstün Y, Aslan T, Şekerci AE, Sağsen B. Evaluation of the reliability of cone-beam computed tomography scanning and electronic apex locator measurements in working length determination of teeth with large periapical lesions. J Endod 2016;42(9):1334-7. 
9. Yılmaz F, Kamburoğlu K, Şenel B. Endodontic working length measurement using cone-beam computed tomographic images obtained at different voxel sizes and field of view, periapical radiography, and apex locator: a comparative ex vivo study. J Endod 2017;43(1):152-6.

10. de Morais ALG, de Alencar AHG, Estrela CR, Decurcio DA, Estrela C. Working length determination using cone-beam computed tomography, periapical radiography and electronic apex locator in teeth with apical periodontitis: A clinical study. Iran Endod J 2016;11(3):164-8.

11. Ghule KD, Naik S. Comparing the accuracy of cone-beam computed tomog raphy and electronic apex locator for root canal length determination in primary teeth. J Indian Soc Pedod Prev Dent 2019;37(2):157-61.

12. Mrasori S, Budina R, Dragidella F. In vitro evaluation of three electronic apex locators using conventional methods and cone beam computed tomography. Minerva Stomatol 2014;63(7-8):259-66.

13. Dutta K, Desai PD, Das UK, Sarkar S. Comparative evaluation of three methods to measure working length - manual tactile sensation, digital radiograph, and multidetector computed tomography: An in vitro study. J Conserv Dent 2017;20(2):76-80.

14. Lucena C, López JM, Martín JA, Robles V, González-Rodríguez MP. Accuracy of working length measurement: Electronic apex locator versus conebeam computed tomography. Int Endod J 2014;47(3):246-56.
15. Metska ME, Liem VML, Parsa A, Koolstra JH, Wesselink PR, Ozok AR. Cone-beam computed tomographic scans in comparison with periapical radiographs for root canal length measurement: An in situ study. J Endod 2014;40(8):1206-9.

16. Aktan AM, Yildirim C, Karataşlıoğlu E, Çiftçi ME, Aksoy F. Effects of voxel size and resolution on the accuracy of endodontic length measurement using cone beam computed tomography. Ann Anat 2016;208:96-102.

17. Connert T, Hülber JM, Godt A, Löst C, ElAyouti A. Accuracy of endodontic working length determination using cone beam computed tomography. Int Endod J 2014;47(7):698-703.

18. Tsesis I, Blazer T, Ben-Izhack G, Taschieri S, Del Fabbro M, Corbella S, et al. The precision of electronic apex locators in working length determination: A systematic review and meta-analysis of the literature. J Endod 2015;41(11):1818-23.

19. Amin J, Lines J, Milosevic MP, Park A, Sholapurkar A. Comparison of accuracy and reliability of working length determination using cone beam computed tomography and electronic apex locator: a systematic review. J Contemp Dent Pract 2019;20(9):1118-23. 